\title{
HARA: Aplicativo com Realidade Aumentada para Apoiar o Ensino de Hardwares de Computadores
}

\author{
Flaviana L. Cruz ${ }^{1}$, Alexandre F. de Castro ${ }^{1}$, Lucas P.C Tavares ${ }^{1}$, Genarde M. \\ Trindade $^{1,2}$, Elisângela S. de Oliveira ${ }^{1}$, Dayane R. de Souza ${ }^{1}$ \\ ${ }^{1}$ Centro de Estudos Superiores de Itacoatiara (CESIT) \\ Universidade do Estado do Amazonas (UEA) \\ Itacoatiara - AM- Brasil \\ ${ }^{2}$ Mestrado em Tecnologias Emergentes em Educação (MTEE) \\ Miami University of Science and Technology (MUST) \\ Boca Raton- FL-Estados Unidos \\ \{flc.lic16, afc.lic17, lpct.lic17, @uea.edu.br, \{genardemacedo, \\ zange07, dayanerosas\}@gmail.com
}

\begin{abstract}
This article describes the development and validation of a mobile application in Augmented Reality for high school students of the technical course in Computer Maintenance and Support at the Federal Institute of Amazonas IFAM Itacoatiara-AM. The study aimed to develop and validate an Augmented Reality application to support the teaching process of computer hardware components the research methodology based on qualitative experimental studies. The results show evidence that the Application can be useful to assist in the process of teaching computer hardware.
\end{abstract}

Resumo. Este artigo descreve o desenvolvimento e validação de um aplicativo móbile em Realidade Aumentada para alunos do ensino médio do curso técnico em Manutenção e Suporte de Computadores do Instituto Federal do Amazonas IFAM Itacoatiara-AM. O estudo objetivou-se a desenvolver $e$ validar um aplicativo em Realidade Aumentada para apoiar o processo de ensino de componentes de hardware de computadores. A metodologia da pesquisa é baseada em estudos experimentais de caráter qualitativo. Os resultados mostram indicios que o Aplicativo pode ser útil para auxiliar no processo de ensino de hardwares de computadores.

\section{Introdução}

Com a evolução das Tecnologias Digitais de Informação e Comunicação (TDICs) tem-se notado mudanças e progressos nos métodos tradicionais de ensino, fazendo-se necessário à utilização de novas tecnologias que possam facilitar o processo de instrução dos conteúdos. [Trindade 2018]. A Realidade Aumentada (RA) tem sido utilizada como ferramenta pedagógica para auxiliar os processos de ensino, pois promove a inserção de objetos virtuais no ambiente real [Kirner e Kirner 2011]. 
Neste contexto, o presente trabalho apresenta o desenvolvimento e a validação tecnológica de um aplicativo com base na tecnologia de RA, denominado HARA, que visa auxiliar o ensino nos conteúdos referentes aos hardwares de computadores, em uma turma do curso técnico em Suporte e Manutenção de Computadores no Campus do Instituto Federal do Amazonas IFAM Itacoatiara-AM.

A metodologia adotada na pesquisa é experimental de caráter qualitativo adaptada parcialmente de [de Souza et al. 2017] e compreende quatro etapas: (1) Revisão da Literatura; (2) Proposta Inicial; (3) Desenvolvimento do Aplicativo; e (4) Validação do Aplicativo.

(1) Revisão da Literatura: Buscou-se subsídios teóricos sobre os assuntos tratados na pesquisa, realizou-se buscas de artigos científicos que possuíam características com a proposta desta pesquisa no Google Scholar e nos principais anais de eventos nacionais na área de Informática na Educação, promovidos com o apoio da Sociedade Brasileira de Computação (SBC), as Strings utilizadas na busca foram: Realidade Aumentada; Aplicativos para educação; desenvolvimento de Software educacional.

(2) Proposta Inicial: Utilizou-se a técnica de entrevista semiestruturada para elicitação de requisitos com o Professor de Informática dos alunos que participariam do experimento na instituição. Analisando as necessidades especificas de ensino para o curso técnico em Manutenção e Suporte de Computadores.

(3) Desenvolvimento do Aplicativo: Para a construção utilizou-se as seguintes ferramentas: (a) Unity 3D, onde executou-se a implementação por meio das linguagens $C \# \mathrm{e}$ JavaScript; (b) Vuforia, realizou-se integração dos marcadores e do banco de dados para execução da RA; (c) Photoshop e Canva, usados para o designer da interface do HARA, bem como a criação dos marcadores. Essas ferramentas tecnológicas foram utilizadas visando atender os requisitos do aplicativo.

(4) Validação do aplicativo: Primeiramente realizou-se o experimento com o profissional da área específica, a fim de verificar se a tecnologia atendia os requisitos necessários de um aplicativo educativo, após realizou-se o experimento com os alunos do curso técnico em Suporte e Manutenção de Computadores. A validação do aplicativo ocorreu por meio de interação com a tecnologia proposta. Para melhor compreensão dos resultados, aplicou-se uma entrevista semiestruturada como o professor de informática e questionários e estudo de observação com os alunos.

\section{Descrição do Aplicativo HARA}

O aplicativo HARA foi desenvolvido por acadêmicos do curso de Licenciatura em Computação do Centro de Estudos Superiores de Itacoatiara (CESIT) da Universidade do Estado do Amazonas (UEA), como proposta na disciplina Análise e Projetos de Sistemas e usando a interdisciplinaridade com a disciplina de Estagio Supervisionado II.

$\mathrm{O}$ aplicativo possibilita que os usuários visualizem objetos bidimensionais (2D). $\mathrm{O}$ HARA pode ser acessado por meio de um smartphone ou tablet que utilize o sistema operacional Android e que esteja com o aplicativo instalado. Para acessar o HARA não há 
necessidade de realizar login, a ferramenta pode ser utilizada para auxiliar nas atividades de ensino de hardwares de computadores. A Figura 1 apresenta as principais telas do HARA.
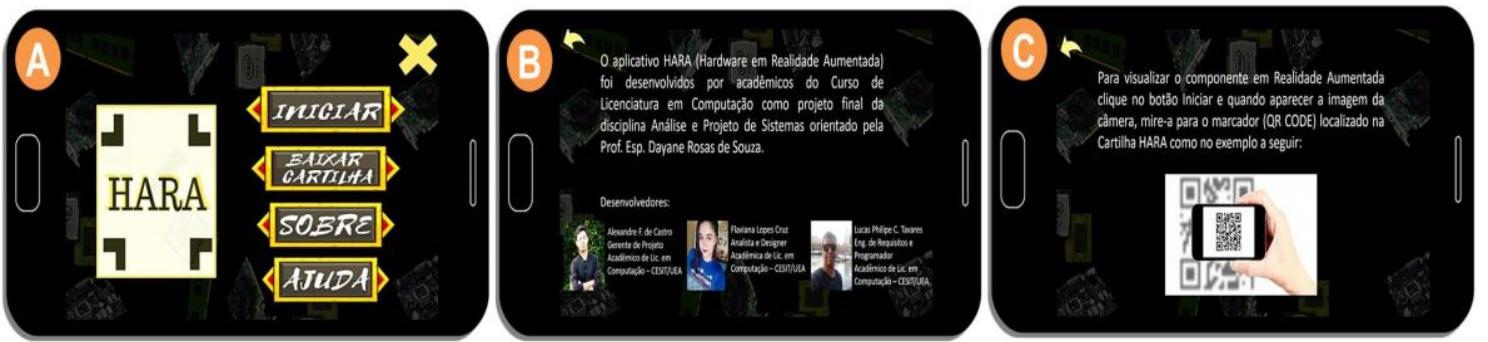

Figura 1. (A) tela Inicial. (B) Tela Sobre. (C) Tela de Ajuda - Fonte: dos autores

A tela inicial na Figura1(A) exibe algumas funções, onde o usuário pode escolher qual a opção desejada, como: a) Botão Iniciar, onde ativa a câmera de RA para ver o objeto virtual sobreposto em ambiente real e preciso colocar o marcador diante da câmera do smartphone ou tablet; b) Botão Baixar Cartilha, onde poderá fazer download dos marcadores por meio do Google Driver e realizar a impressão dos mesmos; c) Botão Sobre, contém informações do aplicativo e dos desenvolvedores, (Figura 1 (B)); d) Botão Ajuda, disponibiliza instruções de uso do HARA, (Figura 1 (C)); e) Botão Sair, opção de sair do aplicativo.

A Figura 2 apresenta alguns objetos 2D sobreposto em RA. Na Figura 2 (A) mostra a memória principal de um computador, na Figura 2 (B) apresenta o disco rígido e a Figura 2 (C) mostra a memória principal de um computador. Todos os objetos virtuais contêm uma breve descrição de cada componente.
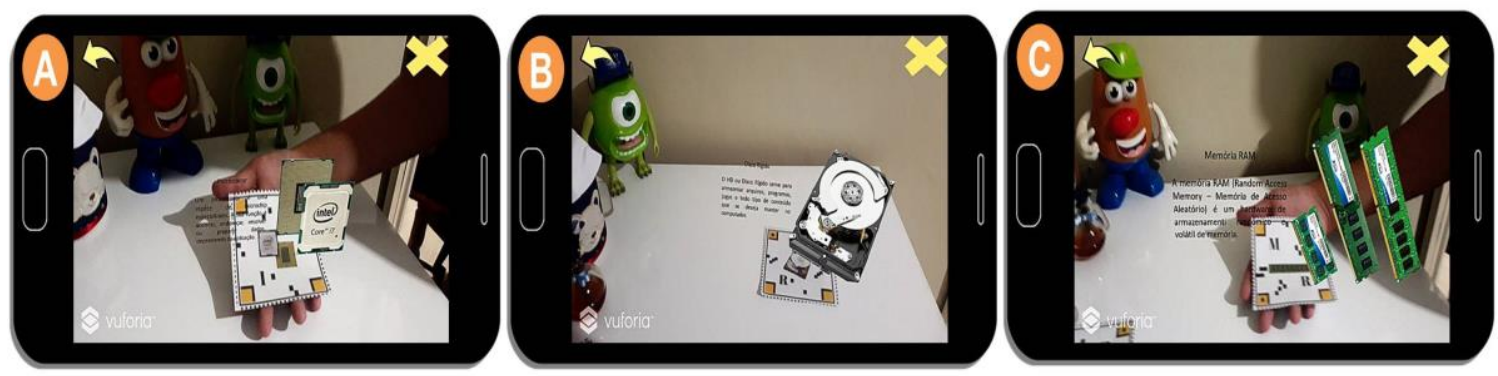

Figura 2. (A) Processador. (B) Disco rígido. (C) Memória principal - Fonte: dos autores

\section{Validação do Aplicativo HARA}

Esta seção apresenta os dois estudos experimentais realizados, a fim de validar o aplicativo HARA. Primeiramente aplicou-se o experimento com o Professor de Informática e o segundo experimento com 15 alunos do curso técnico em Suporte e Manutenção de Computadores.

\subsection{Validação do HARA com profess or}

O objetivo era avaliar se o aplicativo HARA estava adequado para utilização com ferramenta de apoio ao ensino de hardwares de computadores, ou identificar a necessidade de adequações. Dessa forma, a pergunta a ser respondida pelo stakeholder foi " $O$ aplicativo está adequado para o ensino de hardwares de computadores?". Assim, usou-se os seguintes passos para validar o aplicativo com o professor: 
(1) Planejamento: Planejou-se realizar uma entrevista semiestruturada com o stakeholder, na sala dos professores na instituição onde-se aplicou-se o estudo com a duração prevista de uma hora, planejou-se ainda levar o protótipo evolutivo, bem como os marcadores, um smartphone contendo a aplicativo instalado para análise do professor.

(2) Execução do Estudo: O professor interagiu com o HARA, realizando comentários positivos em relação à interface do aplicativo, como: "As cores do aplicativo estão combinando o tamanho das fontes e dos botões estão bons para os alunos". Porém sugeriu que houvesse a inserção de mais marcadores e mais objetos virtuais "Seria bom se pudessem colocar todos os objetos da placa mãe e objetos de circuitos".

(3) Análise dos Resultados: Diante da análise para validação do HARA o professor relatou que a aplicação pode ser utilizada como ferramenta no ensino de hardwares de computadores, sendo necessário a inserção de mais objetos virtuais.

(4) Melhorias: Com base no primeiro experimento, realizou-se a inserção de mais três marcadores e mais três objetos virtuais no aplicativo HARA. Após a implementação dos objetos aplicou-se o experimento com os alunos.

\subsection{Validação do HARA com os alunos}

No segundo estudo avaliou-se a interação dos alunos com a solução proposta, a fim de verificar se o HARA seria utilizado de maneira esperada e se poderia contribuir no ensino de hardwares de computadores. Neste sentido, a pergunta a ser respondida foi " $O$ aplicativo está adequado para conhecer os componentes de computadores?'. Para isso, adotou-se os seguintes passos:

(1) Planejamento: Elaborou-se os seguintes documentos: (a) Termo de Consentimento Livre e Esclarecido (TCLE); (b) Formulário de Caracterização do Participante que continha questões sobre o conhecimento e experiências em aplicativos educacionais; (c) Questionário pós-teste baseado no Modelo de Aceitação Tecnológica (TAM) [Davis. et al 1989]. A proposta do TAM é determinar a facilidade de uso e utilidade de aplicativos educacionais. No questionário TAM os participantes informaram o grau de concordância sobre a utilidade e a facilidade de uso do HARA.

(2) Execução do Estudo: Realizou-se em uma sala da instituição. Primeiramente explicou-se os procedimentos da pesquisa, os alunos receberam e assinaram o TCLE. Após, foi entregue o Formulário de Caracterização e em seguida disponibilizou-se o executável do aplicativo para que os alunos realizassem a instalação nos smartphones e após distribui-se os marcadores. $\mathrm{O}$ estudo teve a duração de 30 minutos e todos interagiram individualmente com o HARA, conforme a Figura 3.
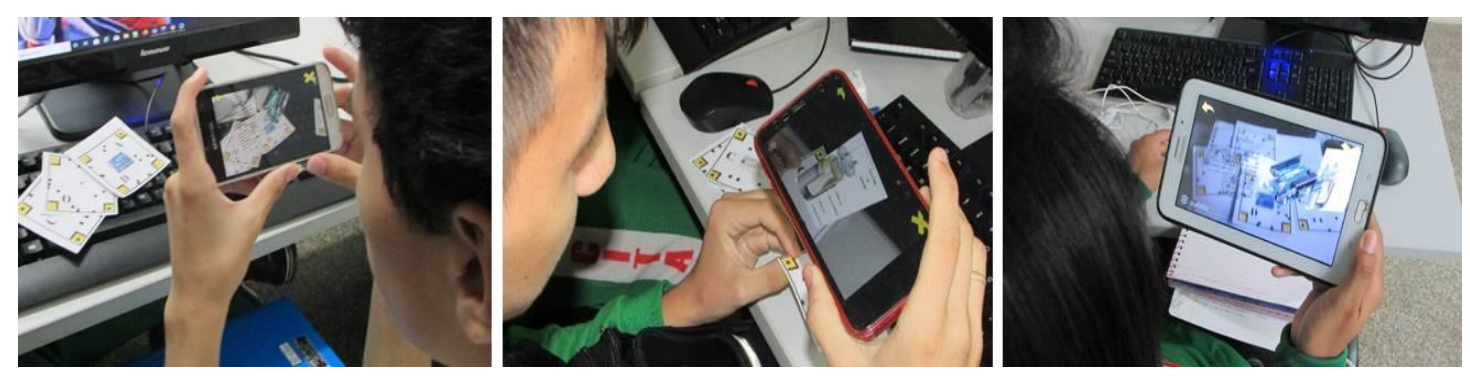
Figura 3. Participante interagindo no aplicativo HARA - Fonte: dos autores

(3) Análise dos Resultados: Os gráficos a seguir mensuram os dados coletados de quinze participantes sobre a facilidade e utilidade do aplicativo HARA.
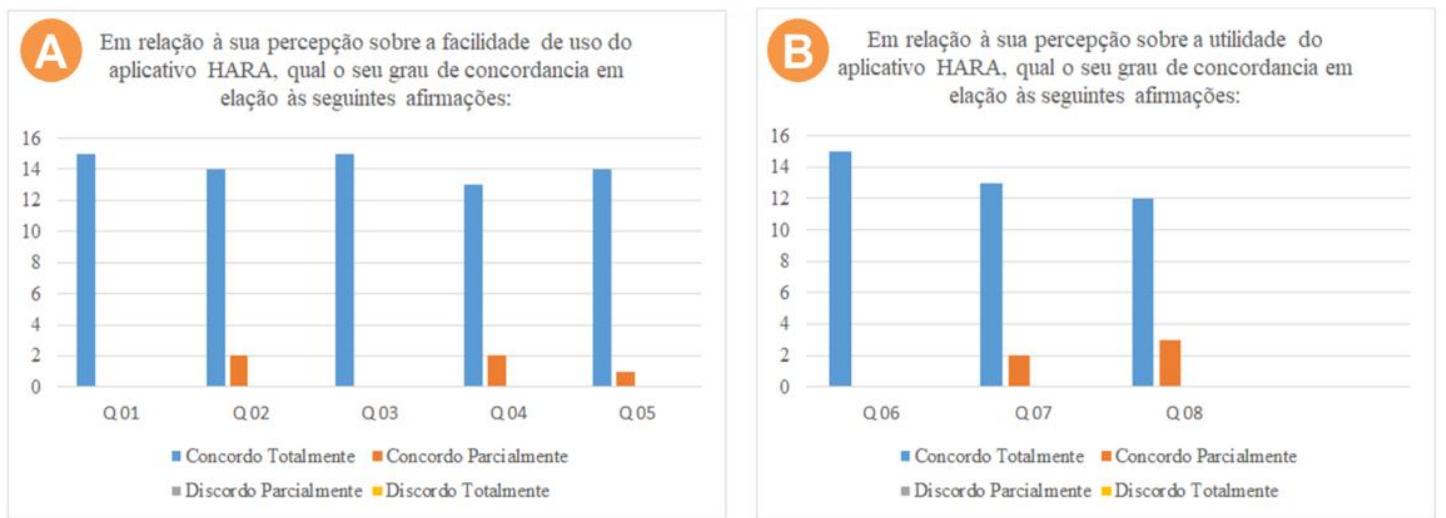

Figura 4. (A) Gráfico sobre a facilidade do HARA. (B) Gráfico sobre o uso do HARA Fonte: dos autores

O gráfico da Figura 4 (A) ilustra a percepção de facilidade de uso do HARA. Assim analisou-se o grau de concordância em relação às seguintes questões: Q01 "Foi fácil aprender a utilizar o HARA?", os quinze participantes concordaram totalmente; Q02 "Consegui utilizar o HARA da forma que eu queria?", treze alunos concordaram totalmente e dois concordaram parcialmente; Q03 "Eu entendia o que acontecia na minha interação com o HARA?", os quinze participantes concordam totalmente; Q04 "Foi fácil ganhar habilidade do uso do HARA?", treze participantes concordam totalmente e dois concorda parcialmente; Q05 “Considero o HARA fácil de usar?”, quatorze participantes concordam totalmente e um concorda parcialmente.

O gráfico da Figura 4 (B) ilustra a percepção sobre a utilidade do HARA. Dessa forma, analisou-se o grau de concordância em relação às seguintes questões: Q06 “O HARA pode ser utilizado no ensino de hardwares de computadores?", os quinze participantes concordam totalmente; Q07 "O aplicativo fornece conteúdo e contribuição útil para o ensino?", treze participantes concordam totalmente e dois concordam parcialmente; Q08 "Indicaria o HARA para outra pessoa?", doze participantes concordam totalmente e três concordam parcialmente.

(4) Lições Aprendidas: Durante o estudo de observação, identificou-se que o Participante 4 apresentou dificuldades na captura de um marcador, após a orientação obteve a visualização do objeto em RA. O participante 7 sugeriu, adicionar um fundo para visualizar melhor as descrições dos objetos virtuais. A maioria dos participantes fizeram comentários positivos sobre o aplicativo, tais como: "Foi bem fácil usar o aplicativo", "Gostei das cores usadas", "O aplicativo não ocupa muito espaço no celular". Assim, identificamos que o aplicativo HARA está adequado para apoiar o ensino de hardwares de computadores. Entretanto, é necessário que haja algumas melhorias para potencializar ainda mais o aplicativo, como por exemplo, incrementar atividades para analisar o aprendizado do aluno e inserir mais objetos virtuais. 


\section{Conclusão e Perspectivas Futuras}

Este artigo apresentou o desenvolvimento e validação de um aplicativo RA, denominado HARA. A Validação foi realizada com um Professor de Informática e posteriormente com quinze alunos do $3^{\circ}$ ano do ensino médio do curso técnico em Manutenção e Suporte de Computadores do Instituto Federal do Amazonas-IFAM campos Itacoatiara-AM. O objetivo do aplicativo HARA é apoiar o ensino de hardwares de computadores. Os resultados do estudo mostram indícios que aplicativo HARA pode ser empregado como ferramenta pedagógica.

Como sugestão de trabalhos futuros, pode-se definir: (1) A inserção de novos objetos com modelagem em 3D; (2) Incremento de novas funcionalidades tais como: Quiz, jogo da memória; (3) Corrigir a apresentação das descrições dos objetos; (4) Realizar um novo estudo com uma amostra maior; (5) Realizar um estudo sobre o processo de ensinoaprendizagem por meio do aplicativo HARA.

\section{Referências}

Davis, F. et al. (1989). "User acceptance of computer technology: a comparison of two theoretical models". Management science.

De Souza, Dayane et al. (2017). “alfabetzAR: Uma Aplicação Móvel com base na Realidade Aumentada como Ferramenta de Apoio no Processo de Alfabetização de Portadores de Síndrome de Down”. In: Brazilian Symposium on Computers in Education (Simpósio Brasileiro de Informática na Educação-SBIE).

Kirner, Claudio; Kirner, Tereza Gonçalves. (2011). "Evolução e tendências da Realidade Virtual e da Realidade Aumentada". Realidade Virtual e Aumentada: Aplicações e Tendências.

Trindade, Genarde Macedo. (2018). "Uma aplicação móvel de realidade aumentada como ferramenta de apoio ao ensino sobre as mesorregiões do Estado do Amazonas". 